\title{
Semi-empirical prediction of physical properties of (B3) TIP compound
}

\author{
Salah Daoud $^{1 *}$, Nadhira Bioud ${ }^{2}$ \\ ${ }^{1}$ Faculté des Sciences et de la Technologie, Université de Bordj Bou Arreridj, 34000, Algeria \\ ${ }^{2}$ Laboratoire d'Optoélectronique et Composants, Université Ferhat Abbes- Sétif, 19000, Algeria \\ *Corresponding author E-mail: salah_daoud07@yahoo.fr
} Copyright $\odot 2014$ Salah Daoud and Nadhira Bioud. This is an open access article distributed under the Creative Commons Attribution License, which permits
unrestricted use, distribution, and reproduction in any medium, provided the original work is properly cited.

\begin{abstract}
The principal goal of this work is the prediction only by means of some emperical formulas and two other physical quantities (the bond length and the bulk modulus calculated initially from first-principle calculations) of the refractive index, the exciton Bohr parameter, the electronic polarizability, the plasmon energy, the force constants, the optical band gap energy, the homopolar and heteropolar energies, the bonding-antibonding energy gap, the ionicity, the dielectric constant, and the optical susceptibility of (B3) thallium phosphide (TIP) compound. In addition, the Debye temperature, the melting temperature, the thermal conductivity and finally the linear thermal expansion of this compound are also obtained. Our results are in general in good agreement with the previous results of the literature.
\end{abstract}

Keywords: Optical Properties, Thermal Properties, TlP Compound.

\section{Introduction}

The physical properties of crystalline solids (which have highly ordered three-dimensional arrangements of atoms) are considerably affected by the chemical constituents (atoms, ions, ...etc) and the symmetry of the crystalline lattice (relative position of atoms in the solid). In recent years, much attention has been given to predict several physical properties of many binary tetrahedral semiconductors ( $\mathrm{SiC}$ compound, $\mathrm{A}^{\mathrm{III}}-\mathrm{B}^{\mathrm{V}}$ group semiconductor materials,...etc) because of their importance in several applications, such as linear and nonlinear optics, light-emitting diodes, etc... [1]. The optoelectronic devices based on thallium-V compounds have some advantages compared to other $\mathrm{A}^{\mathrm{II}}-\mathrm{B}^{\mathrm{VI}}$ semiconductor devices, for many interesting applications, and especially in optical communication systems in the nearinfrared wavelength region [2]. The structural stability of thallium-V compounds: TIX (X=N, P, As, Sb, and Bi) was studied in detail by Saidi-Houat et al. [3]; they found that the cubic zincblende (B3) structure is the more stable compared to other phases for the thallium phosphide compound. Some unusual features, compared with the other $\mathrm{A}^{\mathrm{III}}$ $\mathrm{B}^{\mathrm{V}}$ families, are registered by Saidi-Houat et al. [3] for the TIX compounds.

Using the first-principles plane-wave pesudopotential (PW-PP) approach; the effect of the hydrostatic pressure on the structural phase transition, electronic and elastic properties in three TlX (X = N, P, and As) compounds has been studied in detail by Shi et al [4]; and they found the inexistence of the optical band gap at zero-pressure for all these compounds, exhibiting nearly semi-metallic character [4].

In the cubic common diamond-structure and cubic zincblende structure materials, there are four molecules in the conventional unit cell; each molecule is formed from two atoms. Usually, for the conventional semiconductor materials in these structures, all the atoms are tetrahedrally bonded through covalent bonds to four nearest-neighbor atoms [5].

In the present work, we report the numerical calculations (by using of simple emperical formulas, and two other parameters: the bond length and the bulk modulus which are obtained previously from first principle calculations [6]) of the refractive index, the exciton Bohr parameter, the electronic polarizability, the plasmon energy, the force constants, the optical band gap energy, the homopolar and heteropolar energies, the bonding-antibonding energy gap, the ionicity, the dielectric constant, the optical susceptibility, the Debye temperature, the melting temperature, the linear thermal expansion and finally the thermal conductivity at equilibrium volume of (TIP) compound in its (B3) structure. 


\section{Theory, results and calculations}

\subsection{Refractive index, exciton Bohr parameter and electronic polarizability}

Relationships between the refractive index and a several physical quantities for the $\mathrm{A}^{\mathrm{II}}-\mathrm{B}^{\mathrm{VI}}$ and $\mathrm{A}^{\mathrm{III}}-\mathrm{B}^{\mathrm{V}}$ semiconductor groups have been proposed Reddy et al. [1]. These relationships can be used for the prediction of these quantities for other binary $\mathrm{A}^{\mathrm{III}}-\mathrm{B}^{\mathrm{V}}$ compounds. The refractive index $n$, versus the bond length $d$ and versus the bulk modulus $B$ for some $\mathrm{A}^{\mathrm{II}}-\mathrm{B}^{\mathrm{VI}}$ and $\mathrm{A}^{\mathrm{III}}-\mathrm{B}^{\mathrm{V}}$ semiconductors can be given by the two following relations [1].

$d(\AA)=K_{1} \exp \left(K_{2} n\right)$

$B(G P a)=K_{3} \exp \left(K_{4} n\right)-K_{5}$

The numerical values of the different constants $K_{1}, K_{2}, K_{3}, K_{4}$ and $K_{5}$ for $\mathrm{A}^{\mathrm{III}}-\mathrm{B}^{\mathrm{V}}$ group are respectively: $1.159,0.2364$, $648.89,-0.3546$, and 130.33 [1].

Reciprocally to the formulas of Eqs. (1) and (2), we can obtain two other relationships which can be used to estimate the refractive index $n$ of others binary $\mathrm{A}^{\mathrm{III}}-\mathrm{B}^{\mathrm{V}}$ compounds, by means of the bond length and the bulk modulus of the material, they are given approximately by the following expressions:

$n=K_{2}^{-1} \cdot \ln \left(d / K_{1}\right)$

$n=K_{4}^{-1} \cdot \ln \left[\left(B+K_{5}\right) / K_{3}\right]$

The results of the refractive index $n$, obtained from the relations of Eqs. (3-a) and (3-b), and by using the value ( $2.582 \AA)$ of the bond length and the value (58.08GPa) of the bulk modulus obtained previously from Ab-initio study [6] are: 3.39 and 3.49, respectively.

The exciton Bohr parameter $\left(a_{B}\right)$ ( $1^{\text {st }}$-orbital Bohr radius), which is a size parameter versus the refractive index $n$ for some binary $\mathrm{A}^{\mathrm{III}}-\mathrm{B}^{\mathrm{V}}$ semiconductor compounds, was given as [7]:

$a_{B}=(n / B)^{\beta}$

Where: $B$ and $\beta$ are constants; the numerical values of the constants $B$ and $\beta$ for $\mathrm{A}^{\mathrm{III}}-\mathrm{B}^{\mathrm{V}}$ group are: 5.422 and 5.152 respectively [7]. It is worth noting, that the value 5.422 of $B$, obtained in previous work [7] seems to be inaccurate (wrong), because the later authors used another formula between the dielectric constant $\varepsilon_{1}$ and the exciton Bohr parameter $\left(a_{B}\right)$ which was given as: $a_{B}=\left(\varepsilon_{1} / C\right)^{\delta}$; the numerical values of the constants $C$ and $\delta$ for $\mathrm{A}^{\mathrm{III}}-\mathrm{B}^{\mathrm{V}}$ group are: 1.556 and 2.576, respectively [7]. And because: $\varepsilon_{1}=n^{2}$, we can obtain: $\beta=2 \delta$, and $B=\sqrt{C} \approx 1.2474$ (so it isn't 5.422). By using the relations of Eq. (4) and the values (3.39 and 3.49) of the refractive index, the results obtained of the exciton Bohr parameter $\left(a_{B}\right)$ are: $167.47 \AA$ and $200.45 \AA$ respectively.

Unfortunately, as far as we know, there are no data available in the literature on the refractive index $n$ and on the exciton Bohr parameter $\left(a_{B}\right)$ for (B3) TIP compound. Future experimental work and other theoretical calculations will contribute for the validating of our calculated results of the refractive index $n$ and the exciton Bohr parameter $\left(a_{B}\right)$ for (B3) TIP compound.

The electronic polarizability $\alpha_{\mathrm{p}}$ can be obtained by using the phenomenological approach of Clausius and Mossotti which is given by the following formula [8]:

$\alpha_{p}=\frac{\left(n^{2}-1\right) \cdot M \cdot 10^{-24}}{\left(n^{2}+2\right) \cdot g \cdot(2.53)}$

Where, $M$ is the molecular weight (for TIP, $M=235.357$ uma), $g$ is the crystal density $\left(7.373 \mathrm{~g} / \mathrm{cm}^{3}[6]\right)$ and $n$ is refractive index. The results for: the electronic polarizability $\alpha_{\mathrm{p}}$ obtained by using the refractive index $n$ calculated from the relations of equations (7) and (8) are: $9.81 \AA^{3}$ and $9.95 \AA^{3}$, respectively. These two values of $\alpha_{\mathrm{p}}$ are relatively much higher than the value $\left(7.42 \AA^{3}\right)$ obtained by Verma et al [9].

\section{2. Micro-hardness, plasmon energy, force constants and ionicity}

In this part of this work, we have using a number of equations which have been proposed by Reddy et al. [1] to estimate, the microhardness $H$, the Plasmon energy $\hbar \omega_{p}$, the force constants $(\alpha$ and $\beta)$ and the ionicity $f_{i}$ of TIP compound. The refractive index, versus microhardness, Plasmon energy and force constants for some groups $A^{\mathrm{II}}-\mathrm{B}^{\mathrm{VI}}$ and $A^{\mathrm{III}}-\mathrm{B}^{\mathrm{V}}$ semiconductors can be given by the following relations. [1]

$H(G P a)=K_{6} \exp \left(K_{7} n\right)-K_{8}$

$\hbar \omega_{p}(e V)=K_{9} \exp \left(K_{10} n\right)$

$\alpha(N / m)=K_{11} \exp \left(K_{12} n\right)$ 
$\beta(N / m)=0.28\left(1-f_{i}\right) \alpha$

The relevant values of the different constants $K_{6}, K_{7}, K_{8}, K_{9}, K_{10}, K_{11}$, and $K_{12}$ for $\mathrm{A}^{\mathrm{III}}{ }_{-} \mathrm{B}^{\mathrm{V}}$ group are: 104.953, -0.3546 , $26.82,47.924,-0.3546,286.3$ and -0.6028 , respectively [1].

For the semiconductors of $\mathrm{A}^{\mathrm{III}}-\mathrm{B}^{\mathrm{V}}$ group, the ionicity can be obtained with the following formula [10]:

$f_{i}=K_{13}-K_{14}\left(\hbar \omega_{p}\right)$

The relevant values of the different constants $K_{13}$ and $K_{14}$ for $\mathrm{A}^{\mathrm{III}} \mathrm{B}^{\mathrm{V}}$ groups are: 0.1809 and -0.0126 , respectively [10]. The results for: the microhardness, Plasmon energy, force constants, and ionicity are given in table 1. Unfortunately, as far as we know, there is no data available in the literature on the microhardness, Plasmon energy, and ionicity for (B3) TIP compound. From the table 1, it is seen that, our calculated values of the force constants are relatively lower than the previous calculated data [11].

Table 1: Micro-hardness, Plasmon Energy, Force Constants and Ionicity (B3) TIP: ${ }^{a}$ Using $n=3.39,{ }^{\mathrm{b}}$ Using $n=3.49$.

\begin{tabular}{cccc}
\hline Parameter & \multicolumn{2}{c}{ Our work } & Other works \\
\hline$H(\mathrm{GPa})$ & $4.73^{\mathrm{a}}$ & $3.63^{\mathrm{b}}$ & \\
$\hbar \omega_{p}(\mathrm{eV})$ & $14.40^{\mathrm{a}}$ & $13.90^{\mathrm{b}}$ & $41.21[11]$ \\
$\alpha(\mathrm{N} / \mathrm{m})$ & $37.10^{\mathrm{a}}$ & $34.93^{\mathrm{b}}$ & $7.38[11]$ \\
$\beta(\mathrm{N} / \mathrm{m})$ & $6.62^{\mathrm{a}}$ & $6.30^{\mathrm{b}}$ & \\
$f_{i}$ & $0.36^{\mathrm{a}}$ & $0.36^{\mathrm{b}}$ & \\
\hline
\end{tabular}

\section{3. Band gap energy, homopolar and heteropolar energies, dielectric constant and optical susceptibility}

Knowledge of the optoelectronic properties such as: the refractive index $n$, the extinction coefficient $k$, the optical energy gap $E_{g}, \ldots$ etc, of semiconductor materials is essential to provide valuable information regarding their experimental synthesis and eventual utility in fabricating various devices [4]. In the case of direct transition type semiconductors, the top of the valence band and the bottom of the conduction band are at the same energy axis (direction) and the refractive index of these semiconductor materials are typically decrease with increasing of the band gap energy $E$ g. Anani et al [12] reported that, the refractive index of a semiconductor is dependent on its band gap, and they have proposed a linear relationship between the refractive-index and the band gap energy $E g$, it is given by the following formula:

$n=(17-E g) / 5$

Reciprocally to the formula of Eq. (11), we can obtain the optical band gap energy $E_{g}$ from the following expression:

$E g=17-5 n$

By employing, the value (3.39) of the refractive index obtained from the relation of Eq. (3-a), and with the help of the relation of Equ. (12), the value of band gap energy $E_{g}$ is equal to: $0.05 \mathrm{eV}$. This value shows a small energy gap of thallium-phosphide. This value is much lower comparatively with the theoretical calculation value $(0.158 \mathrm{eV})$ obtained by Wang and Ye [13]. The using of the refractive index value (3.49) obtained from the relation of equation (3-b), give a negative energy gap value, indicating a semi-metallic character of this material. Ciftci et al [2] found also a negative energy gap for the (B3) TIP compound material.

The plasmon energy $\left(\hbar \omega_{p}\right)$, versus the bond length $d$ for some group $\mathrm{A}^{\mathrm{III}} \mathrm{B}^{\mathrm{V}}$ materials, can be given by the following relation [10]:

$d(\AA)=C\left(\hbar \omega_{p}\right)^{-2 / 3}$

The relevant value of the constants $C$ for $\mathrm{A}^{\mathrm{III}} \mathrm{B}^{\mathrm{V}}$ group is equal to: 15.30 [10]. Reciprocally to the relation of Eq. (13), we can obtain approximately the following expression:

$\hbar \omega_{p}=(C / d)^{3 / 2}$

The result for: the plasmon energy, obtained from the relation of equation (14) by using the bond length $d(2.582 \AA$ ) obtained from first principle calculations [6] is equal to: $14.43 \mathrm{eV}$. This value is in excellent agreement with the previous result $(14.41 \mathrm{eV})$ obtained from the application of Eq. (7), and the deviation between the two results is only about $0.14 \%$. The plasmon energy can be also given as function of some others parameters: homopolar energy $\left(E_{h}\right)$, heteropolar energy $\left(E_{c}\right)$, the bonding-antibonding energy gap $\left(E_{g(A-B)}\right)$ and dielectric constant $(\varepsilon)$ by the following relations [10]:

$E_{h}=K_{15}\left(\hbar \omega_{p}\right)^{K 16}$

$E_{c}=K_{17} \cdot \exp \left[K_{18}\left(\hbar \omega_{p}\right)\right]$

$\mathrm{Eg}_{(\mathrm{A}-\mathrm{B})}=-K_{19}+K_{20}\left(\hbar \omega_{p}\right)$

$\varepsilon=K_{21}-K_{22}\left(\hbar \omega_{p}\right)$

The relevant values of the different constants $K_{15}, K_{16}, K_{17}, K_{18}, K_{19}, K_{20}, K_{21}$ and $K_{22}$ for $\mathrm{A}^{\mathrm{III}}-\mathrm{B}^{\mathrm{V}}$ group are taken from the paper of Kumar et al [10]. 
The results for: homopolar energy $\left(E_{h}\right)$, heteropolar energy $\left(E_{c}\right)$, the bonding-antibonding energy gap $\left(E_{g(A-B)}\right)$, and the dielectric constant $(\varepsilon)$, are listed in table 2. Unfortunately, to the best of our knowledge, there is no data available in the literature on these quantities for TIP compound. Thus, further experimental investigations and theoretical predictions are significantly needed.

Table 2: Homopolar Energy, Heteropolar Energy, Bonding-Antibonding Energy $E_{g(A-B}$, and The Dielectric Constant of (B3) TIP: ${ }^{\mathrm{a}}$ Using $\hbar \omega_{p}=14.40 \mathrm{eV},{ }^{\mathrm{b}}$ Using $\hbar \omega_{p}=13.90 \mathrm{eV}$.

\begin{tabular}{llr}
\hline \multicolumn{1}{c}{ Parameter } & \multicolumn{2}{c}{ Our results } \\
\hline$E_{h}(\mathrm{eV})$ & $3.82^{\mathrm{a}}$ & $3.61^{\mathrm{b}}$ \\
$E_{c}(\mathrm{eV})$ & $2.86^{\mathrm{a}}$ & $2.70^{\mathrm{b}}$ \\
$\mathrm{Eg}(\mathrm{A}-\mathrm{B})(\mathrm{eV})$ & $4.67^{\mathrm{a}}$ & $4.28^{\mathrm{b}}$ \\
$\varepsilon$ & $11.48^{\mathrm{a}}$ & $11.95^{\mathrm{b}}$ \\
\hline
\end{tabular}

For object to predict the optical susceptibility $\chi$ of zinc-blende and wurtzite structured binary solids $\left(\mathrm{A}^{\mathrm{III}}-\mathrm{B}^{\mathrm{V}}\right.$ and $\mathrm{A}^{\mathrm{II}}-\mathrm{B}^{\mathrm{VI}}$ group binary materials), Yadav et al. [14] have proposed the following relation.

$\chi=K_{23}\left(\hbar \omega_{p}\right)^{-1.33}$

The relevant value of the constant $K_{23}$, for $\mathrm{A}^{\mathrm{III}}-\mathrm{B}^{\mathrm{V}}$ group is: 27.77 [14].

The proposed empirical relation (19) has been applied to evaluate the optical susceptibility for (B3) TIP compound; the values obtained are equal to: 0.80 (with $\hbar \omega_{p}=14.40 \mathrm{eV}$ ) and 0.84 (with $\hbar \omega_{p}=13.90 \mathrm{eV}$ ) respectively.

\section{4. Debye temperature, melting temperature, thermal conductivity and linear thermal expansion}

The Debye temperature $\left(\theta_{D}\right)$ of semiconductor material is a fundamental thermodynamic parameter, because it correlates directly with many other physical properties: such as specific heat and elastic constants [4]. Experimentally, the Debye temperature can be obtained from the elastic constants measurement method and also from the low temperature specific heat measurement technique.

In recent years, Kumar et al. [15] have proposed a linear relationship between the Debye temperature $\left(\theta_{D}\right)$ and the plasmon energy $\left(\hbar \omega_{p}\right)$, it is given by the following formula:

$\theta_{D}=-K_{24}+K_{25}\left(\hbar \omega_{p}\right)$

For $\mathrm{A}^{\mathrm{III}-} \mathrm{B}^{\mathrm{V}}$ semiconductors, the values of the constants $K_{24}$ and $K_{25}$ are: 800.88 and 77.48 , respectively [15]. Using Eq. (20), the values obtained of $\theta_{\mathrm{D}}$ are: $315.16 \mathrm{~K}$ and $276.28 \mathrm{~K}$, respectively; they have been listed in table 3 and compared with the available theoretical data $\left[2,4,6\right.$, and 16]. It can be seen that, our values of $\theta_{\mathrm{D}}$ lie in the range of data reported in Refs. [2, 4, 6, and 16]. Moreover, our value $(315.16 \mathrm{~K})$ of the $\theta_{\mathrm{D}}$ is in good agreement with the earlier calculations; its deviation is about $4.36 \%$ from the value calculated by Ciftci et al [2].

one of the most essential thermophysical quantities of solid material is its melting temperature; with simple definition: it's the temperature at which it changes state from solid to liquid. Another linear relation between the melting temperature $T_{m}$ and the plasmon energy $\left(\hbar \omega_{p}\right)$ was proposed by Kumar et al [15], it is given by the following formula:

$T m=-K_{26}+K_{27}\left(\hbar \omega_{p}\right)$

The values of the constants $K_{26}$ and $K_{27}$ are respectively: 1604.02 and 204.70 for $\mathrm{A}^{\mathrm{III}}-\mathrm{B}^{\mathrm{V}}$ semiconductors [15].

Using eq. (21), the obtained values of $T_{m}$ for (B3) TIP compound at equilibrium lattice parameter are: $1239.26 \mathrm{~K}$ and 1344.53 K respectively, they are listed in table 3 and compared with the available theoretical data [2], [4].

It is worth noting, that the value $1697 \pm 300 \mathrm{~K}$ of melting temperature obtained in previous work [2], seems to be inaccurate, because the later authors used the value $\left(\mathrm{C}_{11}=94.63 \mathrm{GPa}\right.$ so $\left.0.9463 \mathrm{Mbar}\right)$ to estimate the value of $T m$, and if we use this value $\left(\mathrm{C}_{11}=0.9463 \mathrm{Mbar}\right)$ in the empirical relation $\left(\mathrm{Tm}=553+591 / \mathrm{Mbar} \mathrm{C}_{11} \pm 300 \mathrm{~K}\right)$, we obtain the result: $T m=1112.26 \pm 300 \mathrm{~K}$, which is much lower than the value $1697 \pm 300 \mathrm{~K}$.

Table 3: Debye Temperature $\left(\theta_{D}\right)$ and Melting Temperature $T_{m}$ of (B3) TIP In Comparison With Other Theoretical Values [2], [4], [6], and [16]. ${ }^{\mathrm{a}}$ Using $\hbar \omega_{p}=14.40 \mathrm{eV},{ }^{\mathrm{b}}$ Using $\hbar \omega_{p}=13.90 \mathrm{eV}$.

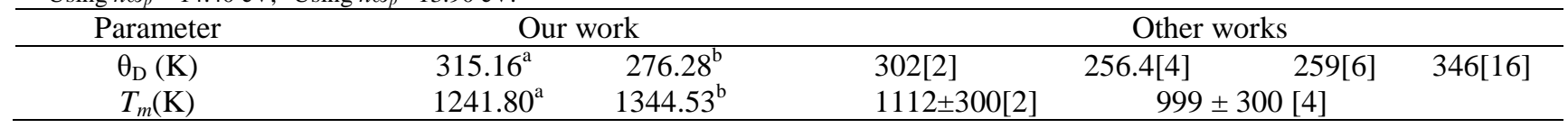

It is interesting to note that, the knowledge of the thermal conductivity $K$ of semiconductor materials is essential and it forms an important part in the design of power-dissipating devices, such as transistors, diodes and other optoelectronic devices [17]. It is important to note also that, the knowledge of the numerical values of $K$ is necessary in calculating the figure of merit for thermoelectronic devices [17]. At $\mathrm{T}=300 \mathrm{~K}$, the thermal conductivity $K$ (in W/cm.K) versus scaling 
parameter $\bar{M} a \theta_{D}^{3}\left(\bar{M} a \theta_{D}^{3}\right.$ in amu.cm. $\left.\mathrm{K}^{3}\right)$ for some group-IV, $\mathrm{A}^{\mathrm{III}-} \mathrm{B}^{\mathrm{V}}$ and $\mathrm{A}^{\mathrm{II}-} \mathrm{B}^{\mathrm{VI}}$ semiconductors can be given by the following relation [17]:

$$
K=1.17 \times 10^{-3}\left(\bar{M} a \theta_{D}^{3}\right)^{1.15}
$$

Where $\bar{M}$ is the average mass of an atom in the crystal (in this case we can take $\bar{M}=M / 2$ ), $M$ is the molecular mass per formula unit, $a$ is the lattice constant and $\theta_{D}$ is the Debye temperature.

For some $\mathrm{A}^{\mathrm{II}} \mathrm{B}^{\mathrm{VI}}$ and $\mathrm{A}^{\mathrm{III}}-\mathrm{B}^{\mathrm{V}}$ binary tetrahedral semiconductors, the lattice thermal conductivity may be evaluated using their ionic charge $\left(Z_{a}\right.$ and $\left.Z_{c}\right)$, and the bond length $d$, all those quantities are related by the following relation [18]:

$K=K_{28}\left(Z_{a} Z_{c}\right)^{K 29} / d^{5}$

Where: $K_{28}$ and $K_{29}$ are constants, which depends up on crystal structure; they have values of 2 and 1.5 , respectively, for zinc-blende structured solids [18].

Our calculated values of the thermal conductivity $K$ for (B3) TIP compound are estimated at about: $0.577 \mathrm{~W} / \mathrm{cm}$.K and $0.47 \mathrm{~W} / \mathrm{cm} . \mathrm{K}$ respectively. They are listed in table 3 and compared with the available theoretical data [18]. Our result $(0.577 \mathrm{~W} / \mathrm{cm} . \mathrm{K})$ is in very good agreement with the previous value $(0.56 \mathrm{~W} / \mathrm{cm} . \mathrm{K})$ obtained by Verma et al [18]. Hence, it is deviating from that $(0.56 \mathrm{~W} / \mathrm{cm} . \mathrm{K})$ of Verma et al [18] only by the difference of $3.04 \%$.

Table 4: Thermal Conductivity of (B3) TIP in Comparison With Other Theoretical Value [18]. ${ }^{\mathrm{a}}$ Using Eq. (22), ${ }^{\mathrm{b}}$ Using Eq. (23).

\begin{tabular}{cccc}
\hline Parameter & Our work & Other work \\
\hline$K(\mathrm{~W} / \mathrm{cm} . \mathrm{K})$ & $0.577^{\mathrm{a}}$ & $0.47^{\mathrm{b}}$ & $0.56[18]$ \\
\hline
\end{tabular}

The linear thermal expansion coefficient $\alpha_{L}$ and the melting temperature $T_{m}$ for tetrahedral material are related by the following empirical formula [19]:

$\alpha_{L}=\left(A / T_{m}\right)-B\left[d-d_{0}\right]^{3}$

Where: $A$ is constant $(A=0.021)$, and $d$ is the equilibrium bond length. For $\mathrm{A}^{\mathrm{III}}-\mathrm{B}^{\mathrm{V}}$ semiconductors, the values of $B$ and $d_{0}$ are equal to: $10\left(10^{-6} \mathrm{~K}^{-1} \AA^{-3}\right)$ and $1.561 \AA$ respectively [19]. The values of $\alpha_{L}$ have been calculated, the result are estimated at about to: $5.38 \times 10^{-6} \mathrm{~K}^{-1}$ (with: $\hbar \omega_{p}=14.40 \mathrm{eV}$ ) and $6.06 \times 10^{-6} \mathrm{~K}^{-1}$ (with: $\hbar \omega_{p}=13.90 \mathrm{eV}$ ). Unfortunately, as far as we know, there are no data available in the literature on the thermal conductivity and $\alpha_{L}$ for (B3) TIP compound.

\section{Conclusion}

Employing the bond-length and the bulk modulus, with the help of some emperical formulas, the refractive index, the exciton Bohr parameter, the electronic polarizability, the plasmon energy, the force constants, the optical band gap energy, the homopolar and heteropolar energies, the bonding-antibonding energy gap, the ionicity, the dielectric constant, the optical susceptibility and the linear thermal expansion of (B3) TIP compound are predicted. Unfortunately, there is no data available in the literature on the majority of these quantities. Future experimental work or other theoretical calculations will contribute for the validating of our calculated results.

Furthermore, the Debye temperature, the melting temperature and the thermal conductivity of this compound are also obtained; they are in general in good agreement with the previous theoretical data of the literature.

\section{References}

[1] R. R. Reddy, Y. Nazeer Ahammed, P. Abdul Azeem, K. Rama Gopal, B. Sasikala Devi \& T. V. R. Rao, "Dependence of Physical Parameters of Compound Semiconductors on Refractive Index", Defense Science Journal, Vol. 53, No. 3, (2003), pp. 239-248. http://www.publications.drdo.gov.in/ojs/index.php/dsj/article/view/2272.

[2] Y. O. Ciftci, K. Colakoglu \& E. Deligöz, "A First Principles Studies on TIX (X=P, As)", Central European Journal of Physics, Vol. 6, No. 4, (2008), pp. 802-807. http://link.springer.com/article/10.2478\%2Fs11534-008-0109-y. http://dx.doi.org/10.2478/s11534-008-0109-y

[3] N. Saidi-Houat, A. Zaoui \& M. Ferhat, "Structural stability of thallium-V compounds", Journal of Physics: Condensed Matter, Vol. 19, No. 10, (2007), pp. 106221 (18pp). http://iopscience.iop.org/0953-8984/19/10/106221/.

[4] L. Shi, Y. Duan \& L. Qin, "Structural phase transition, electronic and elastic properties in TIX (X = N, P, As) compounds: Pressure-induced effects", Computational Materials Science, Vol.50, (2010), pp. 203-210. www.sciencedirect.com/science/article/pii/S0927025610004532. http://dx.doi.org/10.1016/j.commatsci.2010.07.027.

[5] S. Daoud, N. Bioud, N. Lebgaa \& R. Mezouar "Optoelectronic and thermal properties of boron- bismuth compound", International Journal of Physical Research", Vol. 2, No. 2, (2014), pp. 27-31. DOI:10.14419/ijpr.v2i2.2760.

[6] S. Daoud \& N. Bioud "Structural properties of (B3) TlP under pressure", International Journal of Physical Research", Vol. 2, No. 2, (2014), pp. 50-55. DOI: 10.14419/ijpr.v2i2.3100.

[7] N. Korti-baghdadli, A. E. Merad \& T. Benouaz, "Adjusted Adashi's Model of Exciton Bohr Parameter and New Proposed Models for Optical Properties of III-V Semiconductors", American Journal of Materials Science and Technology, Vol.3, (2013), pp. 65-73. DOI:10.7726/ajmst.2013.1008.

[8] A. S. Verma, "Electronic and Optical Properties of Rare-earth Chalcogenides and Pnictides", African Physical Review, Vol.3, (2009), pp. 1120. www.aphysrev.org/index.php/aphysrev/article/download/.../151.

[9] A. S. Verma, R. K. Singh \& S. K. Rathi, "An empirical model for dielectric constant and electronic polarizability of binary (A $\left.{ }^{\mathrm{N}} \mathrm{B}^{8-\mathrm{N}}\right)$ and ternary $\left(\mathrm{A}^{\mathrm{N}} \mathrm{B}^{2+\mathrm{N}} \mathrm{C}_{2}{ }^{7-\mathrm{N}}\right.$ ) tetrahedral semiconductors", Journal of Alloys and Compounds, Vol. 486, No. 1-2, (2009), pp. 795-800. DOI:10.1016/j.jallcom.2009.07.067. 
[10] V. Kumar, A. K. Shrivastava, Anita Sinha \& Vijeta Jha, "Dielectric properties of different materials", Indian Journal of Pure and Applied Physics, Vol. 51, No. 1, (2013), pp. 49-54. nopr.niscair.res.in/bitstream/123456789/../1/IJPAP\%2051(1)\%2049-54.pdf .

[11] A. S. Verma, "Bond-stretching and bond-bending force constant of binary tetrahedral $\left(\mathrm{A}^{\mathrm{III}} \mathrm{B}^{\mathrm{V}}\right.$ and $\left.\mathrm{A}^{\mathrm{II}} \mathrm{B}^{\mathrm{VI}}\right)$ semiconductors", Physics Letters $\mathrm{A}$ Vol. 372, (2008), pp. 7196 -7198. DOI:10.1016/j.physleta.2008.10.043.

[12] M. Anani, C. Mathieu, S. Lebid, Y. Amar, Z. Chama \& H. Abid, "Model for calculating the refractive index of a III-V semiconductor", Computational Materials Science, Vol.41, No.4, (2008), pp. 570-575. www.sciencedirect.com/science/article/pii/S0927025607001577.

[13] S. Q. Wang \& H. Q. Yet, "Plane-wave pseudopotential study on mechanical and electronic properties for IVand III-V crystalline phases with zinc-blende structure", Physical Review B, Vol.66, (2002), pp. 235111 (7pp). link.aps.org/doi/10.1103/PhysRevB.66.235111.

[14] D. S. Yadav, C. kumar, J. Sigh, Parashuram \& G. Kumar, "Optoelectronic properties of zinc blende and wurtzite structured binary solids", Journal of Engineering and Computer Innovations, Vol. 3, No. 2, (2012), pp. 26-35. http://www.academicjournals.org/journal/JECI/articleabstract/DC4DD528645.

[15] V. Kumar, V. Jha, \& A. K. Shrivastava, "Debye temperature and melting point of II-VI and III-V semiconductors", Crystal Research and Technology, Vol. 45, No. 9, (2010) pp. 920-924. http://www. onlinelibrary.wiley.com/doi/10.1002/crat.201000268/pdf.

[16] S. Singh \& M. Sarwan, "High pressure phase transition and elastic behavior of TlX (X=N, P, As) semiconductors", Journal of Physics and Chemistry of Solids, Vol.74, (2013), pp. 487-495. http://dx.doi.org/10.1016/j.jpcs.2012.11.016.

[17] S. Adachi, "Properties of Group-IV, III-V and II-VI Semiconductors", John Wiley \& Sons, England, (2005). http://dx.doi.org/10.1002/0470090340.

[18] A. S. Verma, B. K. Sarkar \& V. K. Jindal, "Inherent properties of binary tetrahedral semiconductors", Physica B, Vol. 405, (2010), pp. 1737 1739. DOI:10.1016/j.physb.2010.01.029.

[19] H. Neumann, "Trends in the thermal expansion coefficients of the $\mathrm{A}^{\mathrm{I}} \mathrm{B}^{\mathrm{III}} \mathrm{C}_{2}{ }^{\mathrm{VI}}$ and $\mathrm{A}^{\mathrm{II}} \mathrm{B}^{\mathrm{IV}} \mathrm{C}_{2}{ }^{\mathrm{V}}$ chalcopyrite compounds", Kristall und Technik, Vol. 15, No. 7, (1980), pp.849-857. DOI: 10.1002/crat.19800150718. 